\title{
OVERDETERMINED PROBLEMS WITH FRACTIONAL LAPLACIAN
}

\author{
Mouhamed Moustapha Fall ${ }^{1}$ And Sven Jarohs ${ }^{2}$
}

\begin{abstract}
Let $N \geq 1$ and $s \in(0,1)$. In the present work we characterize bounded open sets $\Omega$ with $C^{2}$ boundary (not necessarily connected) for which the following overdetermined problem

$$
\left\{\begin{aligned}
(-\Delta)^{s} u & =f(u) & & \text { in } \Omega \\
u & =0 & & \text { in } \mathbb{R}^{N} \backslash \Omega ; \\
\left(\partial_{\eta}\right)_{s} u & =\text { Const. } & & \text { on } \partial \Omega
\end{aligned}\right.
$$

has a nonnegative and nontrivial solution, where $\eta$ is the outer unit normal vectorfield along $\partial \Omega$ and for $x_{0} \in \partial \Omega$

$$
\left(\partial_{\eta}\right)_{s} u\left(x_{0}\right)=-\lim _{t \rightarrow 0} \frac{u\left(x_{0}-t \eta\left(x_{0}\right)\right)}{t^{s}} .
$$

Under mild assumptions on $f$, we prove that $\Omega$ must be a ball. In the special case $f \equiv 1$, we obtain an extension of Serrin's result in 1971. The fact that $\Omega$ is not assumed to be connected is related to the nonlocal property of the fractional Laplacian. The main ingredients in our proof are maximum principles and the method of moving planes.
\end{abstract}

Mathematics Subject Classification. 35B50, 35N25.

Received May 12, 2014. Revised August 29, 2014

Published online May 20, 2015.

\section{INTRODUCTION}

Let $\Omega \subset \mathbb{R}^{N}, N \geq 2$, be a bounded connected open set with $C^{2}$ boundary. By the method of moving planes, Serrin proved, in his celebrated paper [36], that if there is a solution of the overdetermined problem

$$
\left\{\begin{aligned}
-\Delta u=1 & \text { in } \Omega ; \\
u=0 & \text { on } \partial \Omega ; \\
u>0 & \text { in } \Omega ; \\
\partial_{\eta} u=c & \text { on } \partial \Omega
\end{aligned}\right.
$$

\footnotetext{
Keywords and phrases. Fractional Laplacian, maximum principles, Hopf's Lemma, overdetermined problems.

1 African Institute for Mathematical Sciences of Senegal. Km 2, Route de Joal. BP 1418 Mbour, Senegal. mouhamed.m.fall@aims-senegal.org

2 Goethe-Universität Frankfurt, Institut für Mathematik. Robert-Mayer-Str. 10, 60054 Frankfurt, Germany. jarohs@math.uni-frankfurt.de.
} 
then $\Omega$ must be a ball. The motivations for this problem are both from fluid and solid mechanics. Serrin's argument initiated extensive researches in the field of overdetermined problems and symmetry properties of solutions to partial differential equations, see e.g. $[26,27]$ for applications. The moving plane method was first employed in geometry by Alexandrov in [2] to characterize the sphere as the only embedded closed hypersurfaces with constant mean curvature. Over the years there are methods off moving plane to obtain Serrin's result, see e.g. $[6,40]$ and also [18] which reveal the application of Alexandrov's result in the study of (1.1). In those arguments $\Omega$ were assumed to be connected.

Several works have been devoted to related Serrin's problem. Since a complete list of references cannot be given, we only quote: $[1,4,7,8,10-12,19,20,22,23,25,28,31-34,38,39]$.

In the present work we study an overdeterminded problem involving the fractional Laplacian $(-\Delta)^{s}, s \in(0,1)$. It is defined for $u \in C_{c}^{\infty}\left(\mathbb{R}^{N}\right)$ as

$$
(-\Delta)^{s} u(x):=c_{N, s} P . V \cdot \int_{\mathbb{R}^{N}} \frac{u(x)-u(y)}{|x-y|^{N+2 s}} \mathrm{~d} y,
$$

where

$$
c_{N, s}=s(1-s) 4^{s} \pi^{-N / 2} \frac{\Gamma\left(\frac{N}{2}+s\right)}{\Gamma(2-s)}
$$

is a normalization constant (see e.g. [14]) so that $\left(\widehat{-\Delta)^{s}} u(\xi)=|\xi|^{2 s} \widehat{u}(\xi)\right.$. Due to applications in Physics, Biology and Finance, differential equations involving the fractional Laplacian $(-\Delta)^{s}$ have received growing attention in recent years (see e.g. [14], Introduction, and the references therein) but still much less understood than their non-fractional counterparts. The overdeterminded problem we are interested in is

$$
\text { (*) }\left\{\begin{aligned}
(-\Delta)^{s} u & =1 & & \text { in } \Omega ; \\
u & =0 & & \text { in } \mathbb{R}^{N} \backslash \Omega ; \\
\left(\partial_{\eta}\right)_{s} u & \equiv c & & \text { on } \partial \Omega,
\end{aligned}\right.
$$

where $c$ is a negative constant, $\Omega \subset \mathbb{R}^{N}$ is a bounded open set with $C^{2}$ boundary, $\eta\left(x_{0}\right)$ stands for the outer normal at $x_{0} \in \partial \Omega$ and

$$
\left(\partial_{\eta}\right)_{s} u\left(x_{0}\right):=-\lim _{t \rightarrow 0} \frac{u\left(x_{0}-t \eta\left(x_{0}\right)\right)}{t^{s}} .
$$

We note that solutions to the first two equations in $(*)$ are in $C^{s}(\bar{\Omega})$ (see e.g. [37], Prop. 2.9, or [30], Prop. 1) and therefore the overdetermined Neuman condition make sense pointwise. Our first result is contained in the following

Theorem 1.1. Let $\Omega \subset \mathbb{R}^{N}, N \geq 1$, be a bounded open set with $C^{2}$ boundary. Assume that there is a solution $u \in C^{s}(\bar{\Omega})$ of $(*)$. Then $\Omega=B_{R}\left(x_{0}\right)$, a ball centred at $x_{0}$ with radius $R$. In addition $u(x)=$ $\gamma_{N, s}\left(R^{2}-\left|x-x_{0}\right|^{2}\right)^{s}$ for all $x \in B_{R}\left(x_{0}\right)$, where

$$
\gamma_{N, s}:=\frac{4^{-s} \Gamma\left(\frac{N}{2}\right)}{\Gamma\left(\frac{N}{2}+s\right) \Gamma(1+s)} .
$$

We point out that the precise nonnegative and nontrivial function solving $(*)$ when $\Omega$ is a ball were calculated in [5] (see also [15]). We should mention that the overdetermined problem $(*)$ arises from Euler-Lagrange equations of domain dependent variational problems involving the fractional Laplacian. We refer to [13] where the authors considered the case $N=2, s=1 / 2$ and $\partial \Omega$ of class $C^{\infty}$ and assumed $\Omega$ to be connected. In addition, under the aforementioned assumptions, the authors obtained similar result via the moving plane method. We also mention that the authors in [13] used the fact that the $1 / 2$-Laplacian can be realized locally via a Dirichlet-to-Neumann operator. 
Here we use the moving plane argument only in the fractional framework which is a fundamental difference with respect to [13]. In particular our approach could be feasible for more general types of integro-differential equations. Moreover, we take advantages to the nonlocal structure of the fractional laplacian to construct subsolutions yielding a Hopf lemma (see Lem. 3.3) and a version of Serrin's corner boundary point lemma (see Lem. 4.4). The nonlocal structure of $(-\Delta)^{s}$ forces the Dirichlet condition in $\mathbb{R}^{N} \backslash \Omega$. This is a key property which allows us to prove that $\Omega$ must be connected at some stage during the moving plane process.

The moving plane method have been also employed within the fractional framework in $[3,9,17,21,24,29]$.

Our next result is a generalization of the previous one.

Theorem 1.2. Let $c \in \mathbb{R}$ and $\Omega \subset \mathbb{R}^{N}, N \geq 1$, be a bounded open set with $C^{2}$ boundary. Furthermore, let $f: \mathbb{R} \rightarrow \mathbb{R}$ be locally Lipschitz and assume that there is a function $u \in C^{s}(\bar{\Omega})$, which is nonnegative, nontrivial and satisfies

$$
\left\{\begin{aligned}
(-\Delta)^{s} u & =f(u) & & \text { in } \Omega ; \\
u & =0 & & \text { in } \mathbb{R}^{N} \backslash \Omega ; \\
\left(\partial_{\eta}\right)_{s} u & =c & & \text { on } \partial \Omega .
\end{aligned}\right.
$$

Then $\Omega$ is a ball and $u>0$ in $\Omega$.

The paper is organized as follows. In Section 2 we will give some preliminaries and notation. Dealing with the moving plane method leads to dealing with antisymmetric functions so we will give a proof of Hopf's Lemma and some maximum principles for antisymmetric functions in Section 3. Finally, Section 4 is devoted to the proof of Theorem 1.1 and in Section 5, we prove Theorem 1.2.

\section{DEFinitions AND NOTATION}

Let $N \geq 1$ and $s \in(0,1)$. For $u, v \in H^{s}\left(\mathbb{R}^{N}\right)$, we consider the bilinear form induced by the fractional laplacian:

$$
\mathcal{E}(u, v):=\frac{c_{N, s}}{2} \int_{\mathbb{R}^{N}} \int_{\mathbb{R}^{N}} \frac{(u(x)-u(y))(v(x)-v(y))}{|x-y|^{N+2 s}} \mathrm{~d} x \mathrm{~d} y
$$

Furthermore, let

$$
\mathcal{H}_{0}^{s}(\Omega)=\left\{u \in H^{s}\left(\mathbb{R}^{N}\right): u=0 \text { on } \mathbb{R}^{N} \backslash \Omega\right\},
$$

where $\Omega \subset \mathbb{R}^{N}$ is an arbitrary open set. If $\Omega$ is bounded, we define the first Dirichlet eigenvalue:

$$
\lambda_{1}(\Omega)=\min _{u \in \mathcal{H}_{0}^{s}(\Omega)} \frac{\mathcal{E}(u, u)}{\int_{\Omega} u^{2} \mathrm{~d} x} .
$$

Then we have $\lambda_{1}(\Omega) \geq C_{N, s}|\Omega|^{-\frac{2 s}{N}}$, see e.g. [42], where $C_{N, s}=\frac{N}{2 s}\left|B_{1}(0)\right|^{1+2 s / N} c_{N, s}$ and $c_{N, s}$ is the constant in (1.2).

In the following all equalities involving $(-\Delta)^{s}$ are meant in the weak sense, i.e. for $g \in L^{2}(\Omega)$ we call $u \in \mathcal{D}^{s}(\Omega):=\left\{u: \mathbb{R}^{N} \rightarrow \mathbb{R}\right.$ measurable $: \mathcal{E}(u, \varphi)<\infty$ for all $\left.\varphi \in \mathcal{H}_{0}^{s}(\Omega)\right\}$ a supersolution (subsolution) of

$$
(-\Delta)^{s} u=g \quad \text { in } \Omega,
$$

if for all $\varphi \in \mathcal{H}_{0}^{s}(\Omega), \varphi \geq 0$

$$
\mathcal{E}(u, \varphi) \geq \int_{\Omega} g(x) \varphi(x) \mathrm{d} x \quad\left(\mathcal{E}(u, \varphi) \leq \int_{\Omega} g(x) \varphi(x) \mathrm{d} x\right) .
$$


We call $u \in \mathcal{D}^{s}(\Omega)$ an entire supersolution (subsolution) if additionally we have

$$
u \geq 0 \text { on } \mathbb{R}^{N} \backslash \Omega \quad\left(u \leq 0 \text { on } \mathbb{R}^{N} \backslash \Omega\right) .
$$

The function $u$ is called a solution, if $u$ is an entire supersolution and an entire subsolution.

We note that if $u$ is an entire supersolution of (2.1) then $u_{-}=-\min \{w, 0\} \in \mathcal{H}_{0}^{s}(\Omega)$.

Finally we want to note that if $u \in C^{1,1}(\Omega)$, for some open set $\Omega \subset \mathbb{R}^{N}$, we have that $(-\Delta)^{s} u$ is continuous on $\Omega$ (see e.g. [37], Prop. 2.5). Thus if $u$ has such regularity (2.1) holds pointwise.

The following further notation is used throughout the paper: for $x \in \mathbb{R}^{N}$ and $r>0, B_{r}(x)$ is the open ball centered at $x$ with radius $r$ and $\omega_{N}$ will denote the volume of the $N$-dimensional ball with radius 1 . Moreover, we denote $S^{1}:=\left\{x \in \mathbb{R}^{N}:|x|=1\right\}$. For any subset $M \subset \mathbb{R}^{N}$, we denote by $1_{M}: \mathbb{R}^{N} \rightarrow \mathbb{R}$ the characteristic function of $M$ and $\operatorname{diam}(M)$ the diameter of $M$. The notation $A \subset \subset B, A, B \subset \mathbb{R}^{N}$ means that we have $\bar{A} \subset B$ and $\bar{A}$ is compact and nonempty.

Moreover, $w_{+}=\max \{w, 0\}$ and $w_{-}=-\min \{w, 0\}$ denote the positive and negative part of $w$ resp. For any $M \subset \mathbb{R}^{N},|M|$ denotes the Lebesgue measure of $M$ and for $D, U \subset \mathbb{R}^{N}$ we set

$$
\operatorname{dist}(D, U):=\inf (|x-y|: x \in D, y \in U\} .
$$

If $D=\{x\}$, we simply write $\operatorname{dist}(x, U)$ in place of $\operatorname{dist}(\{x\}, U)$. Finally, we denote for $\Omega \subset \mathbb{R}^{N}$,

$$
\delta(x):=\delta_{\Omega}(x):=\operatorname{dist}\left(x, \mathbb{R}^{N} \backslash \Omega\right)
$$

the distance to the complement of a set $\Omega$. We will omit the subindex $\Omega$, whenever no confusion is possible.

\section{MAXIMUM PRINCIPLES FOR ENTIRE ANTISYMMETRIC SUPERSOLUTIONS}

Due to the fact that we will work with the moving plane method in the following, we will need to prove some results concerning antisymmetric functions. Let $H \subset \mathbb{R}^{N}$ be a halfspace, i.e. for any $\lambda \in \mathbb{R}, e \in S^{1}$ we consider

$$
H:=H_{\lambda, e}:=\left\{x \in \mathbb{R}^{N}: x \cdot e>\lambda\right\} .
$$

Let $\Omega \subset H$ be a bounded open set. Let $T:=\partial H$ and denote by $Q: \mathbb{R}^{N} \rightarrow \mathbb{R}^{N}, x \mapsto \bar{x}$ the reflection of $x$ at $T$, i.e. $\bar{x}=x-2(x \cdot e) e+2 \lambda e$. We will call $u \in \mathcal{D}^{s}(\Omega)$ an entire antisymmetric supersolution of $(-\Delta)^{s} u=g$ in $\Omega$, if $u$ is a supersolution of $(-\Delta)^{s} u=g$ in $\Omega$ and if additionally we have $u \geq 0$ on $H \backslash \Omega$ and $u(\bar{x})=-u(x)$ for all $x \in H$.

Proposition 3.1 (Weak Maximum Principle). Let $H$ be a halfspace and let $\Omega \subset H$ be any open, bounded set, let $c \in L^{\infty}(\Omega)$ be such that $c \leq c_{\infty}<\lambda_{1}(\Omega)$ in $\Omega$ for some $c_{\infty} \geq 0$ and let $g \in L^{2}(\Omega)$ be, such that $g \geq-\kappa$ with

$$
0 \leq \kappa<\frac{\lambda_{1}(\Omega)-c_{\infty}}{|\Omega|^{1 / 2}}
$$

If $u$ is an entire antisymmetric supersolution of

$$
(-\Delta)^{s} u=c(x) u+g(x) \quad \text { in } \Omega
$$

then $\left\|u_{-}\right\|_{L^{2}(\Omega)} \leq \kappa|\Omega|^{1 / 2} /\left(\lambda_{1}(\Omega)-c_{\infty}\right)<1$. In particular, if $\kappa=0$ then $u \geq 0$ almost everywhere in $\Omega$.

Proof. Note that $\varphi:=u_{-} 1_{H} \in \mathcal{H}_{0}^{s}(\Omega)$ and

$$
\begin{aligned}
(u(x)-u(y))(\varphi(x)-\varphi(y))+(\varphi(x)-\varphi(y))^{2} & =-u(x) \varphi(y)-u(y) \varphi(x)-2 \varphi(x) \varphi(y) \\
& =-\varphi(x)(\varphi(y)+u(y))-\varphi(y)(\varphi(x)+u(x)) .
\end{aligned}
$$


Thus we have

$$
\begin{aligned}
\mathcal{E}(u, \varphi) & =-\mathcal{E}(\varphi, \varphi)-c_{N, s} \int_{\mathbb{R}^{N}} \int_{\mathbb{R}^{N}} \frac{\varphi(y)(\varphi(x)+u(x))}{|x-y|^{N+2 s}} \mathrm{~d} x \mathrm{~d} y \\
& =-\mathcal{E}(\varphi, \varphi)-c_{N, s} \int_{H} \int_{H} \frac{\varphi(y) u_{+}(x)}{|x-y|^{N+2 s}}+\frac{\varphi(y) u(\bar{x})}{|\bar{x}-y|^{N+2 s}} \mathrm{~d} x \mathrm{~d} y \\
& \leq-\mathcal{E}(\varphi, \varphi)-c_{N, s} \int_{H} \int_{H} \frac{\varphi(y) u_{+}(x)}{|x-y|^{N+2 s}}-\frac{\varphi(y) u(x)}{|\bar{x}-y|^{N+2 s}} \mathrm{~d} x \mathrm{~d} y \\
& =-\mathcal{E}(\varphi, \varphi)-c_{N, s} \int_{H} \varphi(y) \int_{H} u_{+}(x)\left(\frac{1}{|x-y|^{N+2 s}}-\frac{1}{|\bar{x}-y|^{N+2 s}}\right)+\frac{u_{-}(x)}{|\bar{x}-y|^{N+2 s}} \mathrm{~d} x \mathrm{~d} y \\
& \leq-\mathcal{E}(\varphi, \varphi) .
\end{aligned}
$$

Thus we have

$$
\begin{aligned}
0 & \leq \mathcal{E}(u, \varphi)+\int_{\Omega} c(x) \varphi^{2}(x) \mathrm{d} x-\int_{\Omega} g(x) \varphi(x) \mathrm{d} x \\
& \leq-\mathcal{E}(\varphi, \varphi)+c_{\infty}\|\varphi\|_{L^{2}(\Omega)}^{2}+\kappa \int_{\Omega} \varphi(x) \mathrm{d} x \\
& \leq\left(c_{\infty}-\lambda_{1}(\Omega)\right)\|\varphi\|_{L^{2}(\Omega)}^{2}+\kappa|\Omega|^{1 / 2}\|\varphi\|_{L^{2}(\Omega)} .
\end{aligned}
$$

If $\|\varphi\|_{L^{2}(\Omega)}>1$ we have, since $\|\varphi\|_{L^{2}(\Omega)}<\|\varphi\|_{L^{2}(\Omega)}^{2}$,

$$
\begin{aligned}
0 & \leq\left(c_{\infty}-\lambda_{1}(\Omega)\right)\|\varphi\|_{L^{2}(\Omega)}^{2}+\kappa|\Omega|^{1 / 2}\|\varphi\|_{L^{2}(\Omega)} \\
& <\left(c_{\infty}+\kappa|\Omega|^{1 / 2}-\lambda_{1}(\Omega)\right)\|\varphi\|_{L^{2}(\Omega)} \leq 0
\end{aligned}
$$

since $c_{\infty}-\lambda_{1}(\Omega)+\kappa|\Omega|^{1 / 2}<0$, resulting in a contradiction. Thus we must have $\|\varphi\|_{L^{2}(\Omega)} \leq 1$. In this case we have

$$
0 \leq\left(-\left(\lambda_{1}(\Omega)-c_{\infty}\right)\|\varphi\|_{L^{2}(\Omega)}+\kappa|\Omega|^{1 / 2}\right)\|\varphi\|_{L^{2}(\Omega)},
$$

Thus we must have $\|\varphi\|_{L^{2}(\Omega)} \in\left[0, \kappa|\Omega|^{1 / 2} /\left(\lambda_{1}(\Omega)-c_{\infty}\right)\right]$, finishing the proof.

Remark 3.2. Note that the result also holds if $u$ is an entire supersolution of $(-\Delta)^{s} u=c(x) u+g$ in $\Omega$, where $\Omega \subset \mathbb{R}^{N}$ is an arbitrary open, bounded set. The proof then simplifies, since the inequality (3.2) follows trivially.

For the proof of the following version of Hopf's Lemma, we will need the function $\psi_{B}$ satisfying

$$
(-\Delta)^{s} \psi_{B}=1, \quad \text { in } B
$$

where $B \subset \mathbb{R}^{N}$ is a ball. If $B=B_{r}\left(x_{0}\right)$ for some $r>0$ and $x_{0} \in \mathbb{R}^{N}$ we have (see e.g. [5] or [15])

$$
\psi_{B}(x)=\gamma_{N, s}\left(r^{2}-\left|x-x_{0}\right|^{2}\right)_{+}^{s}, \quad \gamma_{N, s}:=\frac{4^{-s} \Gamma\left(\frac{N}{2}\right)}{\Gamma\left(\frac{N}{2}+s\right) \Gamma(1+s)} .
$$

It was proved in ([3], Lem. 4.3), that if $u$ is a continuous supersolution of $(-\Delta)^{s} u=0$ in some open set $\Omega \subset \mathbb{R}^{N}$, such that $u \equiv 0$ on $\mathbb{R}^{N} \backslash \Omega$, then we have for any outernormal $\eta$ and any $x_{1} \in \partial \Omega$ such that there is an interior ball $B \subset \Omega$ with $x_{1} \in \partial B \cap \partial \Omega$, that $\partial_{\eta} u\left(x_{1}\right)=-\infty$ this was enough to carry over a moving plane argument. Here we will need precise behavior of antisymmetric solutions near some corner points at the boundary. A recent result in [35] states that if $u \in \mathcal{H}_{0}^{s}(\Omega)$ is a solution of $(-\Delta)^{s} u=g$ in $\Omega \subset \mathbb{R}^{N}$, with $g \in L^{\infty}(\Omega)$, then $\frac{u}{\delta^{s}} \in C^{0, \alpha}(\bar{\Omega})$, for some $\alpha \in(0,1)$. For $x_{1} \in \partial \Omega$, it therefore make sense to define

$$
\left(\partial_{\eta}\right)_{s} u\left(x_{1}\right):=-\lim _{t \rightarrow 0^{+}} \frac{u\left(x_{1}-t \eta\left(x_{1}\right)\right)}{t^{s}} .
$$




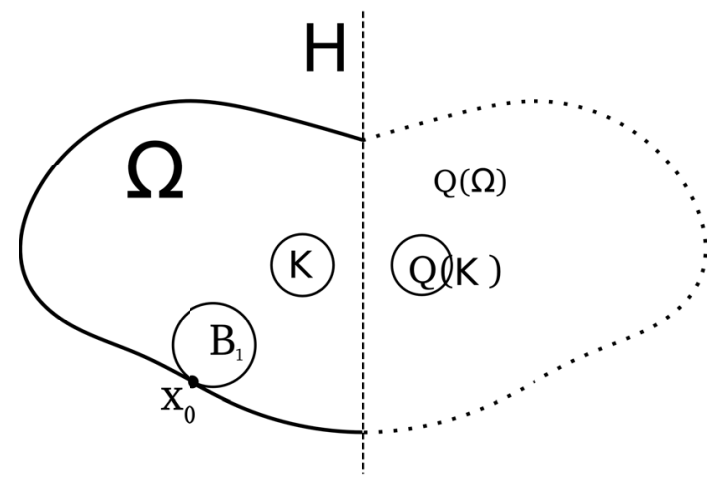

Figure $1 . K$ can be far away from $\Omega$ and does not need to be a ball.

Proposition 3.3 (Hopf's lemma). Let $H \subset \mathbb{R}^{N}$ be a halfspace and $\Omega \subset H$. Consider a ball $B_{1} \subset \subset H$ and $B_{1} \subset \Omega$. Furthermore, let $c \in L^{\infty}(\Omega)$ and assume that $c_{0}=\|c\|_{L^{\infty}(\Omega)}<\lambda_{1}\left(B_{1}\right)$. Let $u \in \mathcal{D}^{s}(\Omega)$ be an entire antisymmetric supersolution of

$$
(-\Delta)^{s} u=c(x) u, \quad \text { in } \Omega \text {; }
$$

such that

$$
u \geq 0, \quad \text { in } H \text {. }
$$

Let $K \subset \subset H \backslash \overline{B_{1}}$ such that $|K|>0$ and suppose that $\operatorname{essinf}_{K} u>0$. Then there is a constant $d=$ $d\left(N, s, \operatorname{diam}\left(B_{1}\right), K, \operatorname{dist}\left(B_{1}, K\right), c_{0}, \operatorname{essinf}_{K} u\right)>0$ such that

$$
u(x) \geq d \delta_{B_{1}}^{s}(x) \quad \text { for almost every } x \in B_{1} .
$$

In particular, if $u \in C\left(\overline{B_{1}}\right)$ and $u\left(x_{0}\right)=0$, for some $x_{0} \in \partial B_{1}$, then we have

$$
-\liminf _{t \rightarrow 0^{+}} \frac{u\left(x_{0}-t \eta\left(x_{0}\right)\right)}{t^{s}}<0 .
$$

Proof. For $\alpha>0$, consider the barrier

$$
w(x):=\psi_{B_{1}}(x)+\alpha 1_{K}(x)-\psi_{Q\left(B_{1}\right)}(x)-\alpha 1_{Q(K)}(x),
$$

where $Q: \mathbb{R}^{N} \rightarrow \mathbb{R}^{N}, x \mapsto \bar{x}$ is the reflection at $\partial H$ and $1_{M}$ is the characteristic function for any $M \subset \mathbb{R}^{N}$. Note that we have $w \in \mathcal{D}^{s}\left(B_{1}\right)$ since $\operatorname{dist}\left(B_{1}, K\right)>0$. Let $\varphi \in \mathcal{H}_{0}^{s}\left(B_{1}\right), \varphi \geq 0$ be arbitrary. Then

$$
\begin{aligned}
\mathcal{E}(w, \varphi)= & \mathcal{E}\left(\psi_{B_{1}}, \varphi\right)+\alpha \mathcal{E}\left(1_{K}, \varphi\right)-\mathcal{E}\left(\psi_{Q\left(B_{1}\right)}, \varphi\right)-\alpha \mathcal{E}\left(1_{Q(K)}, \varphi\right) \\
= & \int_{B_{1}} \varphi(x) \mathrm{d} x-\alpha c_{N, s} \int_{B_{1}} \int_{K} \frac{\varphi(x)}{|x-y|^{N+2 s}} \mathrm{~d} y \mathrm{~d} x \\
& \quad+\alpha c_{N, s} \int_{B_{1}} \int_{Q(K)} \frac{\varphi(x)}{|x-y|^{N+2 s}} \mathrm{~d} y \mathrm{~d} x+c_{N, s} \int_{B_{1}} \int_{Q\left(B_{1}\right)} \frac{\psi_{Q\left(B_{1}\right)}(y) \varphi(x)}{|x-y|^{N+2 s}} \mathrm{~d} y \mathrm{~d} x \\
\leq & \int_{B_{1}} \varphi(x)\left(\kappa-\alpha c_{N, s} \int_{K}\left(|x-y|^{-N-2 s}-|x-\bar{y}|^{-N-2 s}\right) \mathrm{d} y\right) \mathrm{d} x,
\end{aligned}
$$

where $\kappa:=1+\sup _{x \in B_{1}} \psi_{B_{1}}(x) \cdot \sup _{x \in B_{1}, \bar{y} \in H}|x-y|^{-N-2 s}<\infty \operatorname{since} \operatorname{dist}\left(B_{1}, \mathbb{R}^{N} \backslash H\right)>0$. Moreover since $K$ has a positive distance to $\partial H$ and $B_{1}$ we have

$$
C_{1}=C_{1}\left(N, s, K, B_{1}\right)=c_{N, s}|K| \inf _{x \in B_{1}, y \in K}|x-y|^{-N-2 s}-|x-\bar{y}|^{-N-2 s}>0 .
$$


With this we have

$$
\mathcal{E}(w, \varphi) \leq \int_{B_{1}} \varphi(x)\left(\kappa-\alpha C_{1}\right) \mathrm{d} x .
$$

Thus we may take $\alpha$ large so that $\kappa-\alpha C_{1} \leq-c_{0} \sup _{x \in B_{1}} \psi_{B_{1}}(x)$ and thus we have

$$
(-\Delta)^{s} w \leq c(x) w \text { in } B_{1} .
$$

Note furthermore that, by construction, $w=0$ on $\mathbb{R}^{N} \backslash\left(B_{1} \cup K\right)$ and $w(\bar{x})=-w(x)$ for all $x \in \mathbb{R}^{N}$. By assumption, we can pick

$$
\varepsilon=\frac{\operatorname{essinf}_{K} u}{\alpha}>0
$$

so that $v(x):=u(x)-\epsilon w(x) \geq 0$ on $H \backslash B_{1}$. Since we chose $B_{1}$ such that $\|c\|_{L^{\infty}\left(B_{1}\right)}<\lambda_{1}\left(B_{1}\right)$, we can apply Proposition 3.1 to the supersolution $v$ yielding $u \geq \epsilon w=\epsilon \psi_{B_{1}}$ in $B_{1}$. Finally, if $u\left(x_{0}\right)=0$ with $x_{0} \in \partial B_{1}$, we have

$$
-\liminf _{t \rightarrow 0^{+}} \frac{u\left(x_{0}-t \eta\left(x_{0}\right)\right)}{t^{s}} \leq-\epsilon \lim _{t \rightarrow 0^{+}} \frac{\psi_{B_{1}}\left(x_{0}-t \eta\left(x_{0}\right)\right)}{t^{s}}<0 .
$$

As a consequence of Proposition 3.3, we have

Corollary 3.4 (Strong maximum principle). Let $H \subset \mathbb{R}^{N}$ be a halfspace and let $\Omega \subset H$ be an open bounded set. Furthermore let $c \in L^{\infty}(\Omega)$ and $u \in \mathcal{D}^{s}(\Omega)$ be an entire antisymmetric supersolution of

$$
(-\Delta)^{s} u=c(x) u, \quad \text { in } \Omega .
$$

If $u \geq 0$ in $H$ then either $u \equiv 0$ in $H$ or $u>0$ in $\Omega$.

Remark 3.5. We emphasize that Proposition 3.3 remains true for entire supersolutions. Indeed, one would repeat the same proof by considering the barrier $w(x)=\psi_{B_{1}}(x)+\alpha \psi_{K}(x)$. In particular Corollary 3.4 is also valid for entire supersolutions.

\section{Proof of Theorem 1.1}

Our objective in this section is to prove Theorem 1.1 for the case $N \geq 2$. We present an argument based on the fact that $u>0$ in $\Omega$. As we shall see, simple observations shows that after two steps of the moving plane argument, we see that $\Omega$ must be connected. For the case $N \geq 1$ and general right hand side, we postpone the proof in the section.

Theorem 4.1 (Thm. 1.1 for $N \geq 2$ ). Let $\Omega \subset \mathbb{R}^{N}, N \geq 2$, be an open, bounded set such that $\partial \Omega$ is $C^{2}$ and assume that there is a solution $u \in C^{s}(\bar{\Omega})$ of

$$
(-\Delta)^{s} u=1 \quad \text { in } \Omega, \quad u=0 \quad \text { in } \mathbb{R}^{N} \backslash \Omega .
$$

If there is a negative real number $c$ such that

$$
\left(\partial_{\eta}\right)_{s} u \equiv c \quad \text { on } \partial \Omega
$$

then $\Omega$ is a ball and $u=\psi_{\Omega}$, where $\psi_{\Omega}$ is given as in Section 3.

Remark 4.2. We note that by regularity theory $u \in C^{\infty}(\Omega)$. In addition a nontrivial solution $u \in \mathcal{H}_{0}^{s}$ to $(-\Delta)^{s} u=1$ in $\Omega$ is strictly positive in $\Omega$ by Hopf's lemma (see also Rem. 3.5) and there must be $c<0$. 
Proof of Theorem 4.1. Let $e \in S^{1}$ be fixed and consider $T_{\lambda}:=T_{e, \lambda}:=\left\{x \in \mathbb{R}^{N}: x \cdot e=\lambda\right\}$ as a hyperplane in $\mathbb{R}^{N}$, which we will continuously move by continuously varying $\lambda$. Since $\Omega$ is bounded, denote $l:=\max _{x \in \Omega} x \cdot e$, so that $T_{\lambda} \cap \Omega=\emptyset$ for $\lambda \geq l$. Denote $H_{\lambda}:=H_{e, \lambda}:=\left\{x \in \mathbb{R}^{N}: x \cdot e>\lambda\right\}$ and define $\Omega_{\lambda}:=\Omega_{e, \lambda}:=\Omega \cap H_{\lambda}$. Let $Q_{\lambda}:=Q_{e, \lambda}$ be the reflection about $T_{\lambda}$ as described in Section 3 and denote $\Omega_{\lambda}^{\prime}:=Q_{\lambda}\left(\Omega_{\lambda}\right)$, i.e. the reflection of $\Omega_{\lambda}$ about $T_{\lambda}$. Since $\partial \Omega$ is $C^{2}$ we have that for $\lambda<l$ but close to $l$, that $\Omega_{\lambda}^{\prime} \subset \Omega$. As we decrease $\lambda$, i.e. continue moving $T_{\lambda}$, two possible situations may occur:

Situation 1: There is a point $P_{0} \in \partial \Omega \cap \overline{\Omega_{\lambda}^{\prime}} \backslash T_{\lambda}$ or

Situation 2: $T_{\lambda}$ is orthogonal to $\partial \Omega$ at some point $P_{0} \in \partial \Omega \cap T_{\lambda}$.

We note, that although $\Omega$ is not necessarily connected, there is no other possibility since $\partial \Omega$ is $C^{2}$ and $\Omega$ is bounded.

Let $\lambda_{0}$ be the point at which one of these situations occur for the first time.

For simplicity, we put $T=T_{\lambda_{0}}$ and $H=H_{\lambda_{0}}$. Our aim is to prove that if any of the above situation occurs, $\Omega$ must be symmetric with respect to the plane $T$.

To prove that the situations yield symmetry, we let $Q$ be the reflection about $T$ as described in Section 3 . Then define $Q(x)=: \bar{x}$ and consider the function

$$
v(x):=u(x)-u(\bar{x}) \quad \text { for } x \in \mathbb{R}^{N} .
$$

Since $U:=\Omega_{\lambda_{0}}^{\prime} \subset \Omega$ we have that $v$ satisfies

$$
(-\Delta)^{s} v=0 \text { in } U
$$

and

$$
\begin{aligned}
v & \geq 0 & & \text { on } H^{\prime} \backslash U ; \\
v(\bar{x}) & =-v(x) & & \text { for all } x \in \mathbb{R}^{N} .
\end{aligned}
$$

Here $H^{\prime}:=\mathbb{R}^{N} \backslash H$. Thus we have, that $v$ is an entire antisymmetric supersolution on $U$ with $v \geq 0$ on $H^{\prime}$ by the weak maximum principle. The strong maximum principle (Cor. 3.4) then implies $v \equiv 0$ on $\mathbb{R}^{N}$ or $v>0$ in $U$.

We will show, that $v>0$ in $U$ is not possible. This will be separated in to two cases.

Case 1. First assume we are in the first case, i.e. there is some point $P_{0} \in \bar{\Omega} \cap \bar{U} \backslash T$.

Note, that we have $P_{0} \in \partial \Omega \cap \partial U$ due to the choice of $\lambda_{0}$, we have $u\left(P_{0}\right)=0=u\left(\bar{P}_{0}\right)$, especially $v\left(P_{0}\right)=0$. Since $v>0$ in $U$ Hopf's Lemma (Prop. 3.3) gives, that $\left(\partial_{\eta}\right)_{s} v\left(P_{0}\right)<0$, where $\eta$ is the outernormal at $P_{0}$ on $\partial U$. But since $\left(\partial_{\eta}\right)_{s} u\left(P_{0}\right)=c=\left(\partial_{\eta}\right)_{s} u\left(\bar{P}_{0}\right)$ we must have $\left(\partial_{\eta}\right)_{s} v\left(P_{0}\right)=0$ which is a contradiction and thus we cannot be in the first case.

Case 2. Assume that $v>0$ in $U$ and that $T$ is orthogonal to $\partial \Omega$ at a point $P_{0} \in T \cap \partial \Omega$.

Up to translation and rotations, we may assume that $P_{0}=0$ is the origin, $e=e_{1}$, the interior normal of $\partial \Omega$ at the origin is $e_{2}$ and $\nabla^{2} \delta_{\Omega}(0)$ is diagonal. Without loss of generality, we may also assume that $\lambda=0$.

Lemma 4.3. We have

$$
v(t \bar{\eta})=o\left(t^{1+s}\right), \quad \text { as } t \rightarrow 0,
$$

where $\bar{\eta}=(-1,1,0, \ldots, 0)$.

Lemma 4.4. Let $\Omega \subset \mathbb{R}^{N}, N \geq 2$ be an open bounded set with $C^{2}$ boundary such that the origin $0 \in \partial \Omega$. Assume that the hyperplane $\left\{x_{1}=0\right\}$ is orthogonal to $\partial \Omega$ at 0 . Let $D \subset \Omega$ be an open set with $C^{2}$ boundary and 
symmetric about $\left\{x_{1}=0\right\}$. Let $D^{*}:=D \cap\left\{x_{1}<0\right\}$. Let $c \in L^{\infty}\left(D^{*}\right)$ and $w$ be an antisymmetric supersolution of

$$
\begin{aligned}
(-\Delta)^{s} w & \geq c(x) w & & \text { in } D^{*} ; \\
w & \geq 0 & & \text { in }\left\{x_{1}<0\right\} ; \\
w & >0 & & \text { in } D^{*} .
\end{aligned}
$$

Then letting $\bar{\eta}=(-1,1,0 \ldots, 0)$, there exists $C, t_{0}>0$ depending only on $\Omega, N, s$ such that

$$
w(t \bar{\eta}) \geq C t^{1+s} \quad \forall t \in\left(0, t_{0}\right) .
$$

Then applying Lemmas 4.3 and 4.4 (see the proofs below), we reach a contradiction. Therefore $v \equiv 0$ as desired.

If $v \equiv 0$ on $\mathbb{R}^{N}$, then $u \equiv 0$ on $\mathbb{R}^{N} \backslash \tilde{U}$, where $\tilde{U}=U \cup Q_{\lambda_{0}}(U) \cup(T \cap \Omega)$. This implies that $\Omega=\tilde{U}$ yielding symmetry about $T$. It is then clear that $\tilde{U}$ might have many components lined up along $T$.

Assume by contradiction that there are two connected components $\tilde{U}_{1}$ and $\tilde{U}_{2}$.

Observation: There exists a plane $T^{\prime}$ perpendicular to $T$ and separating $\tilde{U}_{1}$ and $\tilde{U}_{2}$. Otherwise one surround the other (recall that they cannot meet at any boundary points by $C^{2}$ regularity of $\partial \tilde{U}$ ) contradicting the minimality of $\lambda_{0}$.

We now move the plane $T^{\prime}$ touching, say, $\tilde{U}_{1}$ first. This leads to property (4.2) with some $\lambda_{1}$ with direction $e^{1}$ and $T^{\prime}=T_{\lambda_{1}}$. Then the same argument as a above yields

$$
\tilde{U}=\tilde{U}_{1}=\tilde{U} \cup Q_{\lambda_{1}}(\tilde{U}) \cup\left(T_{\lambda_{1}} \cap \tilde{U}\right) .
$$

By symmetry of $u$ with respect to $T_{\lambda_{1}}$ and since $u=0$ in $\mathbb{R}^{N} \backslash \tilde{U}_{1}$, we deduce that $u$ vanish in $\tilde{U}_{2}$ which is in contradiction with the fact that $u$ is positive in $\Omega$. Hence $\Omega=\tilde{U}$ is connected with $C^{2}$ boundary.

Restarting the moving plane process, we conclude that $\Omega$ is symmetric with respect to all planes for which Situation 1 and/or Situation 2 occur for a first time. We then conclude that $\Omega$ must be a ball.

We observe that Lemma 4.3 states that "derivatives of order $1+s$ " of $v$ vanish at the origin.

Proof of Lemma 4.3. Thanks to ([35], Thm. 1.2), we can write

$$
u(x)=\delta^{s}(x) \psi(x),
$$

where $\psi(x) \in C^{0, \alpha}(\bar{\Omega})$ for some $\alpha \in(0,1)$ (recall that $\delta=\delta_{\Omega}$ is the distance function to $\partial \Omega$ ). It is clear from our hypothesis that

$$
\psi(x)=-c \quad \forall x \in \partial \Omega .
$$

Put $\bar{u}(x)=u(\bar{x})=u\left(-x_{1}, x_{2}, \ldots, x_{N}\right), \bar{\delta}(x)=\delta(\bar{x})$ and $\bar{\psi}(x)=\psi(\bar{x})$. By continuity, we have

$$
\psi(t \bar{\eta})=-c+o(1)=\bar{\psi}(t \bar{\eta}), \quad \text { as } t \rightarrow 0 .
$$

Then we have

$$
v(t \bar{\eta})=u(t \bar{\eta})-\bar{u}(t \bar{\eta})=\left[\delta^{s}(t \bar{\eta})-\bar{\delta}^{s}(t \bar{\eta})\right](c+o(1)), \quad \text { as } t \rightarrow 0 .
$$

By Taylor expansion, we have

$$
\delta(t \bar{\eta})=\delta(0)+\nabla \delta(0) \cdot(t \bar{\eta})+\frac{1}{2} \nabla^{2} \delta(0)[(t \bar{\eta})] \cdot(t \bar{\eta})+o\left(t^{2}\right), \quad \text { as } t \rightarrow 0
$$




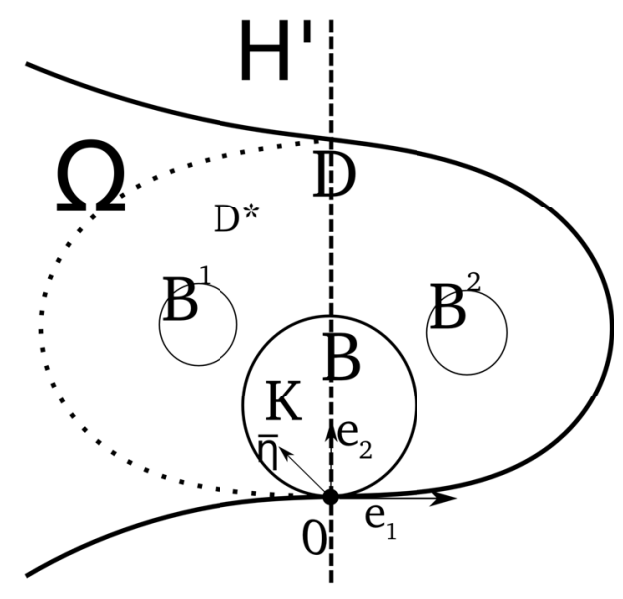

Figure 2. $B^{2}=Q\left(B^{1}\right)$, where $Q$ is the reflection at $\partial H^{\prime}$.

and

$$
\bar{\delta}(t \bar{\eta})=\delta(0)+\nabla \bar{\delta}(0) \cdot(t \bar{\eta})+\frac{1}{2} \nabla^{2} \bar{\delta}(0)[(t \bar{\eta})] \cdot(t \bar{\eta})+o\left(t^{2}\right), \quad \text { as } t \rightarrow 0 .
$$

In addition, since $e_{2}=\nabla \delta(0)$ is the normal direction, $\partial_{x_{i}} \delta(0)=0$ for all $i \neq 2$. Therefore

$$
\nabla \delta(0) \cdot \bar{\eta}=\nabla \bar{\delta}(0) \cdot \bar{\eta}=e_{2} \cdot \bar{\eta}=1 .
$$

Since $\nabla^{2} \delta(0)$ is diagonal, it is plain that

$$
\nabla^{2} \delta(0)[\bar{\eta}] \cdot \bar{\eta}=\nabla^{2} \bar{\delta}(0)[\bar{\eta}] \cdot \bar{\eta}=\nabla^{2} \delta(0)\left[e_{2}\right] \cdot e_{2}+\nabla^{2} \delta(0)\left[e_{1}\right] \cdot e_{1} .
$$

It follows that

$$
\delta^{s}(t \bar{\eta})=t^{s}\left(1+\frac{s}{2} \nabla^{2} \delta(0)[\bar{\eta}] \cdot(t \bar{\eta})+o(t)\right), \quad \text { as } t \rightarrow 0
$$

and

$$
\bar{\delta}^{s}(t \bar{\eta})=t^{s}\left(1+\frac{s}{2} \nabla^{2} \delta(0)[\bar{\eta}] \cdot(t \bar{\eta})+o(t)\right), \quad \text { as } t \rightarrow 0 .
$$

We then conclude that

$$
\delta^{s}(t \bar{\eta})-\bar{\delta}^{s}(t \bar{\eta})=o\left(t^{1+s}\right), \quad \text { as } t \rightarrow 0
$$

This together with (4.5) prove the claim.

We also observe that Lemma 4.4 can be seen as the Serrin's corner boundary point lemma.

Proof of Lemma 4.4. Let $R>0$ small so that $B:=B_{R}\left(R e_{2}\right) \subset \Omega$ and $\partial B_{R}\left(R e_{2}\right) \cap \partial \Omega=\{0\}$. Put

$$
K=B_{R}\left(\operatorname{Re}_{2}\right) \cap\left\{x_{1}<0\right\} .
$$

Define $B^{2}=B_{R}(4 R \eta)$ and $B^{1}=B_{R}(4 R \bar{\eta})$, where $\bar{\eta}=e_{2}-e_{1}$. From now on we will consider $R$ small such that $B^{1} \cup B^{2} \subset \subset D$ (see Fig. 2 below):

We next consider the truncated distance functions to the boundary of these balls denoted by

$$
\mathrm{d}^{2}(x)=(R-|x-4 R \eta|)_{+}, \quad \mathrm{d}^{1}(x)=(R-|x-4 R \bar{\eta}|)_{+} .
$$

As in Section 3 we use

$$
\varphi_{B}(x)=\left(R^{2}-\left|x-R e_{2}\right|^{2}\right)_{+}^{s},
$$


and for $\alpha>0$ (to be chosen later), we consider the barrier

$$
h(x)=-x_{1}\left[\varphi_{R}(x)+\alpha\left(\mathrm{d}^{1}(x)+\mathrm{d}^{2}(x)\right)\right] .
$$

Note that $h(x)=-h(\bar{x})$ and $h \in C^{1,1}(B)$. Using ([15], Thm. $1+$ Tab. 3, p. 549), together with a scaling and translation,

$$
\left|(-\Delta)^{s}\left(x_{1} \varphi_{R}(x)\right)\right|=\left|C_{N, s} R^{-1} x_{1}\right| \leq C\left|x_{1}\right| \quad \forall x \in K,
$$

where here and in the following $C$ is a positive constant (possibly depending on $R, N, s$ ) but never on $\alpha$. Now we put

$$
I(x):=-(-\Delta)^{s}\left[x_{1}\left(\mathrm{~d}^{1}(x)+\mathrm{d}^{2}(x)\right)\right] .
$$

Then for $x \in K$ we have

$$
\begin{aligned}
I(x) & =-P . V . \int_{\mathbb{R}^{N}} \frac{-y_{1}\left(\mathrm{~d}^{1}(y)+\mathrm{d}^{2}(y)\right)}{|x-y|^{N+2 s}} \mathrm{~d} y \\
& =\int_{B^{1}} y_{1} \mathrm{~d}^{1}(y)\left(|x-y|^{-N-2 s}-|x-\bar{y}|^{-N-2 s}\right) \mathrm{d} y \\
& =\int_{B^{1}} y_{1} \mathrm{~d}^{1}(y)|x-y|^{-N-2 s}\left(1-\left(\frac{|x-y|}{|x-\bar{y}|}\right)^{N+2 s}\right) \mathrm{d} y, \\
& =\int_{B^{1}} y_{1} \mathrm{~d}^{1}(y)|x-y|^{-N-2 s}\left(1-\left(\frac{|x-y|^{2}}{|x-y|^{2}+4 x_{1} y_{1}}\right)^{(N+2 s) / 2}\right) \mathrm{d} y .
\end{aligned}
$$

Observe that by construction,

$$
7 R>|x-y|>R \quad \forall x \in \cap B_{R}\left(R e_{2}\right), \quad \forall y \in B^{1} .
$$

Using this and the fact that the map

$$
a \mapsto 1-\left(\frac{a}{a+d}\right)^{k}
$$

is strictly monotone decreasing in $a$ for all $d, k>0$, we therefore get

$$
I(x) \leq \int_{B^{1}} y_{1} \mathrm{~d}^{1}(y)|x-y|^{-N-2 s}\left(1-\left(\frac{(7 R)^{2}}{(7 R)^{2}+4 x_{1} y_{1}}\right)^{(N+2 s) / 2}\right) \mathrm{d} y .
$$

Hence we have (recall that $y_{1}<0$ )

$$
\begin{aligned}
I(x) & \leq-C \int_{B^{1}}\left(1-\left(\frac{(7 R)^{2}}{(7 R)^{2}+4 x_{1} y_{1}}\right)^{(N+2 s) / 2}\right) \mathrm{d} y \\
& =-C \int_{B^{1}}\left(1-\left(1-\frac{4 x_{1} y_{1} /(7 R)^{2}}{1+4 x_{1} y_{1} /(7 R)^{2}}\right)^{(N+2 s) / 2}\right) \mathrm{d} y .
\end{aligned}
$$

Using the elementary inequality

$$
(1-t)^{\beta} \leq 1-t \quad \text { for } t \in(0,1), \beta>1
$$

we get, for all $x \in K$,

$$
I(x) \leq-C \int_{B^{1}} \frac{4 x_{1} y_{1} /(7 R)^{2}}{1+4 x_{1} y_{1} /(7 R)^{2}} \mathrm{~d} y \leq-C\left|x_{1}\right|,
$$


where we have used the fact that $\left|y_{1}\right|$ is bounded away from 0 as long as $y \in B^{1}$ and $\left|x_{1}\right| \leq 5 R$. Combining this with (4.6), we infer

$$
(-\Delta)^{s} h(x)-c(x) h(x) \leq(C-\alpha C)\left|x_{1}\right| \quad \forall x \in K .
$$

Hence we can choose $\alpha$ so that $(-\Delta)^{s} h-c(x) h \leq 0$ in $K$. Since also we can find a positive constant $M>0$ so that $w \geq M h$ in $\overline{B^{1}}$, we get immediately $w-M h \geq 0$ in $\left\{x_{1}<0\right\} \backslash K$. We then deduce from the weak maximum principle that $w \geq M h$ in $D^{*}$. Now since

$$
h(t \bar{\eta})=t^{1+s}\left(2 R-4 t^{2}\right)
$$

the proof follows immediately because $t \bar{\eta} \in D^{*}$ for $t>0$ small.

\section{Generalization}

Theorem 5.1 (Thm. 1.2 for $N \geq 2$ ). Let $c \in \mathbb{R}$ and $\Omega \subset \mathbb{R}^{N}, N \geq 2$, be an open, bounded set with $C^{2}$ boundary. Furthermore, let $f: \mathbb{R} \rightarrow \mathbb{R}$ be locally Lipschitz and assume that there is a function $u \in C^{s}(\bar{\Omega})$, which is nonnegative and nontrivial in $\Omega$ and fulfills

$$
\left\{\begin{aligned}
(-\Delta)^{s} u & =f(u) & & \text { in } \Omega ; \\
u & =0 & & \text { in } \mathbb{R}^{N} \backslash \Omega ; \\
\left(\partial_{\eta}\right)_{s} u & =c & & \text { on } \partial \Omega .
\end{aligned}\right.
$$

Then $\Omega$ is a ball and $u>0$ in $\Omega$.

Proof. Let $e \in S^{1}$ and consider $\lambda_{0}$ as defined in (4.2) and $U:=\Omega_{\lambda_{0}}^{\prime} \subset \Omega$ as before. We define $v_{\lambda_{0}}(x):=$ $u(x)-u(\bar{x})$ for all $x \in \mathbb{R}^{N}$, where we use the notation as usual, i.e. $Q_{\lambda, e}(x)=: \bar{x}$ and $Q_{\lambda, e}$ is the reflection of $T=T_{\lambda, e}$. Then $v_{\lambda_{0}}$ solves

$$
(-\Delta)^{s} v_{\lambda_{0}} \geq-c_{f}(x) v_{\lambda_{0}} \quad \text { in } U
$$

where

$$
c_{f}(x):=\left\{\begin{aligned}
\frac{f(u(x))-f(u(\bar{x}))}{u(x)-u(\bar{x})}, & \text { if } u(x) \neq u(\bar{x}) ; \\
0, & \text { if } u(x)=u(\bar{x}) .
\end{aligned}\right.
$$

Let $L_{f}$ be the Lipschitz constant of $f$ for $\mathcal{B}=\left[0,\|u\|_{L^{\infty}\left(\mathbb{R}^{N}\right)}\right]$. Then we have $\|c\|_{L^{\infty}(U)} \leq L_{f}$. Here, we cannot directly apply the maximum principle to get $v_{\lambda_{0}} \geq 0$ in $H^{\prime}$ as in the previous section because $L_{f}$ might be large. However, by using the moving plane method, we can prove that

$$
v_{\lambda_{0}} \geq 0 \quad \text { on } H^{\prime}
$$

To this end, we observe that for $\lambda \in\left(\lambda_{0}, l\right)$ but close to $l$ we have $L_{f} \leq \lambda_{1}\left(\Omega_{\lambda}^{\prime}\right)$ so that $u(x)-u\left(Q_{\lambda, e}(x)\right) \geq 0$ in $\Omega_{\lambda}^{\prime}$ by the weak maximum principle. Now by the strong maximum principle

$$
\left(S_{\lambda}\right) \quad v_{\lambda}(x):=u(x)-u\left(Q_{\lambda}(x)\right)>0 \quad \text { for all } x \in \Omega_{\lambda}^{\prime}
$$

as $u$ is nontrivial. We let

$$
\tilde{\lambda}:=\inf \left\{\lambda>\lambda_{0}:\left(S_{\mu}\right) \text { holds for all } \lambda>\mu\right\} .
$$

Our aim is to prove that $\tilde{\lambda}=\lambda_{0}$. Assume by contradiction that $\tilde{\lambda}>\lambda_{0}$. Then by continuity and the strong maximum principle we have that $\left(S_{\tilde{\lambda}}\right)$ holds. Since $\tilde{\lambda}>\lambda_{0}$, there is by continuity $\epsilon>0$ such that $\Omega_{\tilde{\lambda}-\epsilon}^{\prime} \subset \Omega$. Choose an open set $\Pi \subset \Omega_{\tilde{\lambda}-\epsilon}^{\prime}$ such that $\{v \leq 0\} \cap \Omega_{\tilde{\lambda}-\epsilon}^{\prime} \subset \Pi$ and we may assume that $|\Pi|$ is small by making $\epsilon$ possibly smaller. The maximum principle then can be applied to $\Pi$ giving $v_{\tilde{\lambda}-\epsilon}>0$ in $\Pi$ (as before) and thus $S_{\tilde{\lambda}-\epsilon}$ holds in contradiction to the choice of $\tilde{\lambda}$. Thus $\tilde{\lambda}=\lambda_{0}$. Hence (5.2) is proved. We have now that $v$ is an 
entire antisymmetric supersolution on $U$ with $v_{\lambda_{0}} \geq 0$ in $H^{\prime}$ by the weak maximum principle. Arguing as in the proof of Theorem 1.1 in the previous section, we obtain $v_{\lambda_{0}} \equiv 0$ in $\mathbb{R}^{N}$. This implies that $u$ is symmetric with respect to all planes $T_{\lambda_{0}, e}=T_{\lambda_{0}(e), e}$ for which (4.1) occur for a first time. More than that, the moving plane process above yield monotonicity through lines perpendicular to $T_{\lambda_{0}, e}$ : for every $e \in S^{1}$

$$
u(x)-u\left(Q_{\lambda, e}(x)\right)>0 \quad \text { for all } \lambda \in\left(\lambda_{0}, l\right) \text { and for all } x \in \Omega_{\lambda, e}^{\prime} .
$$

In particular for all $e \in S^{1}$ and for all $\lambda \in \mathbb{R}$, we have either $u(x) \geq u\left(Q_{\lambda, e}(x)\right)$ for all $x \in H_{\lambda, e}$ or $u(x) \leq$ $u\left(Q_{\lambda, e}(x)\right)$ for all $x \in H_{\lambda, e}$. Now by a well known result (which we include a proof in the Appendix for the readers convenience), $u$ coincides to a radial function up to a translation which is decreasing. Hence $\operatorname{supp}(u)$ is a ball.

We claim that $\bar{\Omega}=\operatorname{supp}(u)$. Indeed, assume on the contrary that $\operatorname{supp}(u) \neq \bar{\Omega}$. Then there is a ball $B \subset \subset$ $\Omega \backslash \operatorname{supp}(u)$, such that $u \equiv 0$ in $B$. Consider the hyperplane $T$ separating $B$ and $\operatorname{supp}(u)$. It is clear that $u \equiv 0$ on the halfspace $H$ with boundary $T$ containing $B$. Let $e \in S^{1}$ normal to $T$ and contained in $H$. Now by moving the planes $T_{\lambda, e}$ as above, we get, for very $\lambda \in\left(\lambda_{0}, l\right)$

$$
u(x)>u\left(Q_{\lambda, e}(x)\right) \geq 0 \quad \text { for all } x \in \Omega_{\lambda, e}^{\prime} .
$$

This, in particular, implies that $u>0$ in $\Omega \cap H_{\lambda_{0}, e}$ which is impossible.

Theorem 5.2 (Thms. 1.1 and 1.2 for $N=1$ ). Let $c \in \mathbb{R}$ and $\Omega \subset \mathbb{R}$ be a bounded open set. Let $f: \mathbb{R} \rightarrow \mathbb{R}$ be locally Lipschitz and assume that there is a function $u \in C(\bar{\Omega})$, which is nonnegative, nontrivial in $\Omega$ and satisfies

$$
\left\{\begin{aligned}
(-\Delta)^{s} u & =f(u) & & \text { in } \Omega ; \\
u & =0 & & \text { in } \mathbb{R} \backslash \Omega ; \\
\left(\partial_{\eta}\right)_{s} u & =c & & \text { on } \partial \Omega .
\end{aligned}\right.
$$

Then $\Omega=(\alpha, \beta)$ for some $\alpha, \beta \in \mathbb{R}, \alpha<\beta$ and $u>0$ in $\Omega$.

Proof. Now assume that $\Omega$ has at least two different connected components $(\alpha, \beta)$ and $(a, b)$ with $a<b<$ $\alpha<\beta$. Note that as in the case $N \geq 2$ we can move points (instead of moving planes!) from the right up to $\lambda_{0}=(\alpha+\beta) / 2$, so that $v(x):=u(x)-u(\bar{x})$ solves

$$
(-\Delta)^{s} v \geq-c_{f}(x) v \quad \text { in }\left(a, \lambda_{0}\right)
$$

and $v(x) \geq 0$ for $x<\lambda_{0}$ by arguing as in the proof of Theorem 5.1. Note that only interior touching can occur. Hence by Hopf's Lemma we obtain $v \equiv 0$ on $\mathbb{R}$, but this gives $u \equiv 0$ on $\mathbb{R} \backslash(\alpha, \beta)$. Next moving from the left up to $\lambda_{0}=(a+b) / 2$ implies, as previously, $u \equiv 0$ in $(a, b)$. Therefore $u \equiv 0$ in $\mathbb{R}$ leading to a contradiction. The positivity of $u$ finally follows as in Theorem 5.1 by the monotonicity which is a byproduct of the moving plane method.

\section{ApPENDIX}

The result below was stated in [41].

Proposition 6.1. Let $u: \mathbb{R}^{N} \rightarrow \mathbb{R}$ be continuous and such that $\lim _{|x| \rightarrow \infty} u(x)=c_{\infty} \in \mathbb{R} \cup\{ \pm \infty\}$ exists. Then the following statements are equivalent:

(i) There is $z \in \mathbb{R}^{N}$, such that $u(\cdot-z)$ or $-u(\cdot-z)$ is radially symmetric and decreasing in the radial variable.

(ii) For every half space $H \in \mathcal{H}$ we either have $u(x)-u\left(Q_{H}(x)\right) \geq 0$ for all $x \in H$ or $u(x)-u\left(Q_{H}(x)\right) \leq 0$ for all $x \in H$. We say $H$ is dominant or subordinate for $u$ respectively. 
Here $\mathcal{H}$ is the set of all affine half spaces in $\mathbb{R}^{N}$ and $Q_{H}$ is the reflection at $\partial H$ for any $H \in \mathcal{H}$.

The following proof is communicated to the authors by Tobias Weth.

Proof. Assertion (i) implies (ii) is obvious and can be found in [41]. Thus we only need to show that (ii) implies (i). Without restriction, we may assume that $u$ is not constant. Consider the halfspace $H_{\lambda}=\left\{x \in \mathbb{R}^{N}: x_{1}>\lambda\right\}$, for $\lambda \in \mathbb{R}$ and the open set

$$
I:=\left\{\lambda \in \mathbb{R}: u(x)>u\left(Q_{H_{\lambda}}(x)\right) \text { for some } x \in H_{\lambda}\right\} .
$$

Since $u$ is not constant, we may assume, replacing $u$ with $-u$ if necessary, that $u$ is larger than $c_{\infty}$ at some point in $\mathbb{R}^{N}$. Hence there exists a maximal $s_{1} \in \mathbb{R} \cup\{\infty\}$ such that $I$ contains the interval $\left(-\infty, s_{1}\right)$. By (ii) the half spaces $H_{\lambda}, \lambda \in\left(-\infty, s_{1}\right)$ are dominant for $u$, which implies that

$$
u \text { is nondecreasing in } x_{1} \text { in the set }\left\{x \in \mathbb{R}^{N}: x_{1}<s_{1}\right\} .
$$

This forces $s_{1}<\infty$, since $u$ is not constant and tends to $c_{\infty}$ as $|x| \rightarrow \infty$. By the maximal choice of $s_{1}$ and the continuity of $u, u$ is symmetric with respect to the hyperplane $\left\{x_{1}=s_{1}\right\}$. By the same argument, we can find $s_{i} \in \mathbb{R}$, such that $u$ is symmetric with respect to the hyperplanes $\left\{x_{i}=s_{i}\right\}$ for $i=2, \ldots, N$. Translating $u$ if necessary, we may assume, that $s_{i}=0$ for $i=1, \ldots, N$, so that $u$ is symmetric with respect to all coordinate reflections. This implies that $u$ is even, i.e. $u(x)=u(-x)$ for all $x \in \mathbb{R}^{N}$. As a consequence, if $H \in \mathcal{H}_{0}=\{H \in$ $\mathcal{H}: 0 \in \partial H\}$ is such that $u(x) \geq u\left(Q_{H}(x)\right)$ for all $x \in H$, then also $u\left(Q_{H}(x)\right)=u\left(-Q_{H}(x)\right) \geq u(-x)=u(x)$ for every $x \in H$, so that $u$ is symmetric with respect to the hyperplane $\partial H$. This implies that $u$ is symmetric with respect to any hyperplane containing 0 , so that $u$ is radially symmetric (see e.g. [41], Sect. 2.1). Finally, (6.1) implies that $u$ is decreasing in the radial variable.

Acknowledgements. The authors would like to thank Tobias Weth for helpful discussions and Xavier Ros-Oton for taking their attention to [15]. Part of this work was done while the second author was visiting AIMS-Senegal. He would like to thank them for their kind hospitalities. The first author is supported by the Alexander von Humboldt foundation.

\section{REFERENCES}

[1] V. Agostiniani and R. Magnanini, Symmetries in an overdetermined problem for the Green's function. Discrete Contin. Dyn. Syst. Ser. S 4 (2011) 791-800.

[2] A.D. Alexandrov, Uniqueness theorems for surfaces in the large I. Vestnik Leningrad Univ. Math. 11 (1956) 5-17.

[3] M. Birkner, J.A. López-Mimbela and A. Wakolbinger, Comparison results and steady states for the Fujita equation with fractional Laplacian. Ann. Inst. Henri Poincaré 22 (2005) 83-97.

[4] I. Birindelli and F. Demengel, Overdetermined problems for some fully non linear operators. Comm. Partial Differ. Eq. 38 (2013) 608-628.

[5] K. Bogdan and T. Byczkowski, Potential Theory of Schrödinger Operator based on fractional Laplacian. Probab. Math. Stat. 20 (2000) 293-335.

[6] B. Brandolini, C. Nitsch, P. Salani and C. Trombetti, Serrin-type overdetermined problems: an alternative proof. Arch. Ration. Mech. Anal. 190 (2008) 267-280.

[7] F. Brock and A. Henrot, A symmetry result for an overdetermined elliptic problem using continuous rearrangement and domain derivative. Rend. Circ. Mat. Palermo 51 (2002) 375-390.

[8] G. Buttazzo and B. Kawohl, Overdetermined boundary value problems for the $\infty$-laplacian. Int. Math. Res. Not. 2011 (2011) $237-247$.

[9] W. Chen, C. Li and Biao Ou, Classification of solutions for an integral equation. Comm. Pure Appl. Math. 59 (2006) 330-343.

[10] M. Choulli and A. Henrot, Use of the domain derivative to prove symmetry results in partial differential equations. Math. Nachr. 192 (1998) 91-103.

[11] A. Cianchi and P. Salani, Overdetermined anisotropic elliptic problems. Math. Ann. 345 (2009) 859-881.

[12] F. Da Lio and B. Sirakov, Symmetry results for viscosity solutions of fully nonlinear uniformly elliptic equations. J. Eur. Math. Soc. (JEMS) 9 (2007) 317-330.

[13] A.-L. Dalibard and D. Gérard-Varet, On shape optimization problems involving the fractional laplacian. ESAIM: COCV 19 (2013) 976-1013. 
[14] E. di Nezza, G. Palatucci and E. Valdinoci, Hitchhiker's Guide to the Fractional Sobolev Spaces. Bull. Sci. Math. 136 (2012) $521-573$.

[15] B. Dyda, Fractional calculus for power functions and eigenvalues of the fractional Laplacian. Fract. Calc. Appl. Anal. 15 (2012) 536-555.

[16] L.C. Evans and R.F. Gariepy, Measure Theory and Fine Properties of Functions. CRC Press, Boca Raton (1992).

[17] M.M. Fall, T. Weth, Monotonicity and nonexistence results for some fractional elliptic problems in the half space. Preprint (2013). Available online at: http://arxiv.org/abs/1309.7230.

[18] A. Farina and B. Kawohl, Remarks on an overdetermined boundary value problem. Calc. Var. Partial Differ. Eq. 31 (2008) 351-357.

[19] A. Farina and E. Valdinoci, Flattening results for elliptic PDEs in unbounded domains with applications to overdetermined problems. Arch. Ration. Mech. Anal. 195 (2010) 1025-1058.

[20] A. Farina and E. Valdinoci, Overdetermined problems in unbounded domains with Lipschitz singularities. Rev. Mat. Iberoam. 26 (2010) 965-974.

[21] P. Felmer, A. Quaas and J. Tan, Positive solutions of Nonlinear Schrödinger equation with the fractional Laplacian. In Vol. 142A, Proc. of Roy. Soc. Edinburgh (2012).

[22] I. Fragalà and F. Gazzola, Partially overdetermined elliptic boundary value problems. J. Differ. Eq. 245 (2009) $1299-1322$.

[23] I. Fragalà, F. Gazzola, and B. Kawohl, Overdetermined problems with possibly degenerate ellipticity, a geometric approach. Math. Z. 254 (2006) 117-132.

[24] P. Felmer and Y. Wang, Radial symmetry of positive solutions involving the fractional Laplacian. Commun. Contemp. Math. (2013). Available at: http://www.worldscientific.com/doi/pdf/10.1142/S0219199713500235.

[25] N. Garofalo and J.L. Lewis, A symmetry result related to some overdetermined boundary value problems. Am. J. Math. 111 (1989) 9-33.

[26] B. Gidas, W.-M. Ni and L. Nirenberg, Symmetry and related problems via the maximum principle. Comm. Math. Phys. 68 (1979) 209-243.

[27] B. Gidas, W.-M. Ni and L. Nirenberg, Symmetry of positive solutions of nonlinear equations. Math. Anal. Appl. Part A, Adv. Math. Suppl. Studies A 7 (1981) 369-402.

[28] L. Hauswirth, F. Hélein and F. Pacard, On an overdetermined elliptic problem. Pacific J. Math. 250 (2011) 319-334.

[29] S. Jarohs, T. Weth, Asymptotic symmetry for a class of fractional reaction-diffusion equations. Discrete Contin. Dyn. Syst. 34 (2014) 2581-2615.

[30] G. Palatucci, O. Savin and E. Valdinoci, Local and global minimizers for a variational energy involving a fractional norm. Ann. Mat. Pura Appl. 192 (2013) 673-718.

[31] J. Prajapat, Serrin's result for domains with a corner or cusp. Duke Math. J. 91 (1998) $29-31$.

[32] A.G. Ramm, Symmetry problem. Proc. Amer. Math. Soc. 141 (2013) 515-521.

[33] W. Reichel, Radial symmetry for an electrostatic, a capillarity and some fully nonlinear overdetermined problems on exterior domains. Z. Anal. Anwendungen 15 (1996) 619-635.

[34] W Reichel, Radial symmetry for elliptic boundary-value problems on exterior domains. Arch. Rational Mech. Anal. 137 (1997) 381-394.

[35] X. Ros-Oton and J. Serra, The Dirichlet Problem for the fractional Laplacian: Regularity up to the boundary. J. Math. Pures Appl. 101 (2014) 275-302.

[36] J. Serrin, A Symmetry Problem in Potential Theory. Arch. Rational Mech. Anal. 43 (1971) 304-318.

[37] L. Silvestre, Regularity of the obstacle problem for a fractional power of the Laplace operator. Comm. Pure Appl. Math. 60 (2007) 67-112.

[38] L. Silvestre and B. Sirakov, Overdetermined problems for fully for fully nonlinear elliptic equations. Preprint (2013) available online at: http://arxiv.org/abs/1306.6673.

[39] B. Sirakov, Symmetry for exterior elliptic problems and two conjectures in potential theory. Ann. Inst. Henri Poincaré Anal. Non Lin. 18 (2001) 135-156.

[40] H.F. Weinberger, Remark on the preceding paper of Serrin. Arch. Rational Mech. Anal. 43 (1971) 319-320.

[41] T. Weth, Symmetry of solutions to variational problems for nonlinear elliptic equations via reflection methods. Jahresber. Deutsch. Math.-Ver. 112 (2010) 119-158.

[42] S.Y. Yolcu, T. Yolcu, Estimates for the sums of eigenvalues of the fractional Laplacian on a bounded domain. Commun. Contemp. Math. 15 (2013) 1250048. 